\title{
Taladrado de pizarra mediante láser de Nd:YAG
}

\author{
M.S. Larosi ${ }^{(*)}$, F. Lusquiños ${ }^{(*)}$, J. Pou ${ }^{(*)}$, R. Soto ${ }^{(*)}$ y M. Pérez-Amor ${ }^{(*)}$
}

Resumen Se ha desarrollado una serie de experimentos para investigar la viabilidad del uso de un láser de Nd:YAG para el procesamiento de piezas de pizarra, así como los parámetros que más afectan al proceso. El objetivo concreto de este trabajo es investigar la capacidad de un láser de Nd:YAG pulsado para taladrar orificios de pequeño diámetro en láminas de pizarra, con el fin de obtener mejor calidad de taladrado en un tiempo reducido. Se presenta parte de los resultados obtenidos en un estudio sistemático de la influencia de los parámetros de procesamiento en la forma del orificio, tales como, potencia, duración de pulso, frecuencia, presión y tipo de gas de aportación.

Palabras clave: Pizarra. Láser. Taladrado.

\section{Nd:YAG laser drilling of slate}

\begin{abstract}
A serie of experiments to investigate the feasibility of using a Nd:YAG laser to process slate tiles and the influence of the processing parameters have been undertaken. The objective of this work is to investigate the capabilities of a Nd:YAG pulsed laser to drill tiny holes in slate tiles, in order to produce a better quality drilled slate tile in a reduced time. Part of the results obtained in a systematic study about the influence of the processing parameters, such as average power, pulse width, frequency, pressure and type of assisting gas is presented.
\end{abstract}

Keywords: Slate. Laser. Drilling.

\section{INTRODUCCIÓN}

La pizarra es una roca homogénea, de grano muy fino, opaca, tenaz y que se puede separar fácilmente en hojas delgadas y planas. Se compone fundamentalmente de cuarzo, clorita, y sericita; es frecuente, además, encontrar óxidos de hierro, sulfuros, sustancias carbonosas y minerales arcillosos. Su textura característica, observada por microscopía óptica, es de aspecto filamentoso con agregados paralelos finos, lo que la hace muy adecuada para obtener elementos de cubrición o pizarras de techar (1).

Solamente en Galicia se pueden distinguir 22 variedades de pizarra, cada una con sus propias características, siendo las más importantes: la densidad $\left(1,5-2,85 \mathrm{~g} / \mathrm{cm}^{3}\right)$, coeficiente de absorción de agua, $(1-3 \%)$, resistencia a la flexión mecánica $\left(300-550 \mathrm{~kg} / \mathrm{cm}^{3}\right)$, resistencia a las heladas, resistencia a los cambios térmicos y resistencia a los áci-

(*) Dpto. Física Aplicada Univ. de Vigo, Lagoas Marcosende 9. 36280-Vigo (España) dos. Para este trabajo, se han seleccionado dos de las más representativas: "El Caurel-Quiroga" y "Valdeorras". La diferencias principales son la resistencia a la flexión mecánica y el contenido de carbonato, más elevado en "El Caurel-Quiroga" (2).

Desde su invención, los láseres han demostrado un potencial enorme en muchos campos (3), transformando las comunicaciones y la medicina. El presente trabajo está orientado hacia el estudio de las capacidades de los láseres para procesar placas de pizarra.

Las principales tareas que deben realizarse para llegar a obtener placas de pizarra para uso en cubiertas son las siguientes: extracción de grandes bloques en la cantera, denominados "rachones"; labrado de los rachones; aserrado de los mismos; exfoliado de las placas y recorte de los tamaños y formas comerciales.

Tradicionalmente, la elaboración de estas placas de pizarra viene realizándose de modo manual, siendo una labor penosa para el trabajador. Se hace, por tanto, necesaria la introducción de nuevos métodos o técnicas que permitan automatizar los procesos y reducir o eliminar completamente la 
emisión de polvo y la producción de ruido, de forma que se favorezcan las condiciones de trabajo.

Como primer paso, se presentan algunos resultados del estudio realizado sobre las posibilidades de taladrar placas de pizarra mediante un láser de Nd:YAG de alta potencia.

\section{PROCEDIMIENTO EXPERIMENTAL}

El taladrado se ha llevado a cabo mediante un láser de Nd:YAG pulsado de $1 \mathrm{~kW}$ de potencia utilizando la técnica del perforado por percusión de pulsos múltiples (4), (en esta técnica no hay movimiento relativo entre el haz láser y la pieza). Como gases auxiliares se han empleado $\mathrm{N}_{2}(99,9 \%)$ y $\mathrm{O}_{2}$ (99,96 - 99,98\%). El sistema de lentes utilizado para focalizar el haz láser sobre la pieza es un doblete acromático de $80 \mathrm{~mm}$ de distancia focal y una ventana de sílice fundida para proteger la lente de posibles choques de las partículas que se desprenden de la pizarra (5).

Con objeto de estudiar sistemáticamente la influencia de los diferentes parámetros de procesamiento en las características de los orificios, se han variado los siguientes parámetros: energía por pulso, potencia, duración de pulso y presión, tanto para nitrógeno como para oxígeno. Debido a la baja adherencia de la escoria expelida del orificio, no ha sido necesario un gas adicional para retirar los restos fundidos de la pieza.

La forma de los orificios ha sido analizada con un micrómetro $X Y$ acoplado a un microscopio óptico para determinar el diámetro del orificio y una cámara $C C D$ para visualizar y almacenar las imágenes del orificio de entrada y de salida.

\section{DISCUSIÓN Y RESULTADOS}

A diferencia de los materiales homogéneos (6), como los metales, muchos tipos de polímeros, cerámicas y materiales no metálicos (7), como la pizarra, presentan dificultades adicionales en la evaluación de los parámetros de procesamiento, debido a su composición y estructura no uniformes.

La figura 1 muestra el número de pulsos que se requieren para perforar completamente una placa de pizarra en función de la energía por pulso, para láminas de pizarra de 2,7, 5 y $12 \mathrm{~mm}$ de espesor. Para pizarras de mayor espesor, se requieren pulsos de una duración mayor que $1 \mathrm{~ms}$, dado que pulsos menores que $1 \mathrm{~ms}$ conducen a un taladrado incompleto debido a la absorción de la energía en las paredes del orificio, después de múltiples reflexiones; lo mismo ocurre al disminuir la presión por debajo de los 4 bar, debido a la acumulación de material fundido en la parte superior de la pieza.

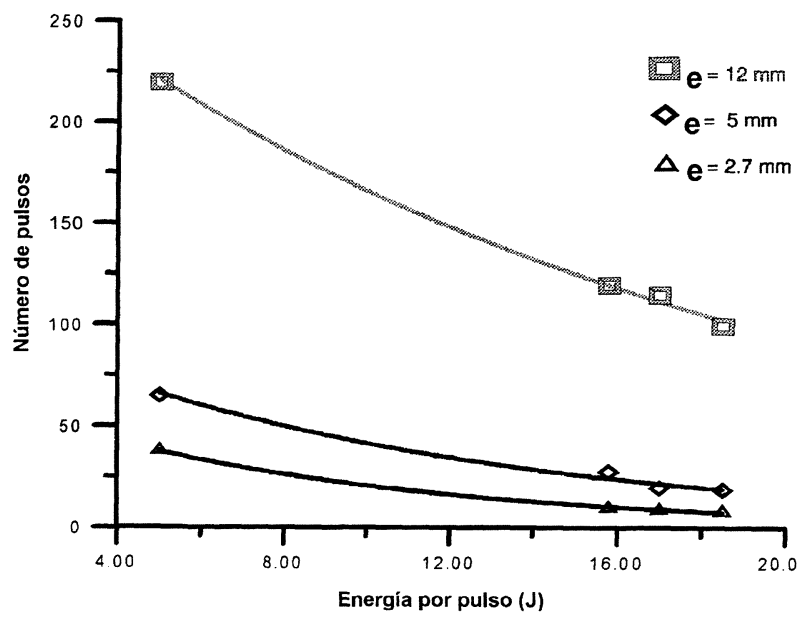

Fig. 1.- Número de pulsos necesarios para realizar un taladrado completo en función de la energía por pulso, para placas de diferentes espesores.

FIG. 1.-Dependence of the number of laser pulses required for complete drilling from pulse energy, for slate plates of different thicknesses.

Para poder estimar la forma del orificio se ha determinado la relación entre el diámetro de entrada y el de salida (8). En la figura 2 se presenta la diferencia entre el diámetro de entrada y el de salida en función de la potencia media, para diferentes presiones de los gases auxiliares utilizados (oxígeno y nitrógeno). Como se puede deducir de la figura, la forma de los orificios se aproxima mucho a la cilíndrica, con cierta conicidad para el caso en el que, como gas auxiliar, se ha utilizado nitrógeno a 6 bar.

En la figura 3 se muestra la influencia de la duración de los pulsos en el diámetro de entrada. En dicha figura, se recopilan los resultados

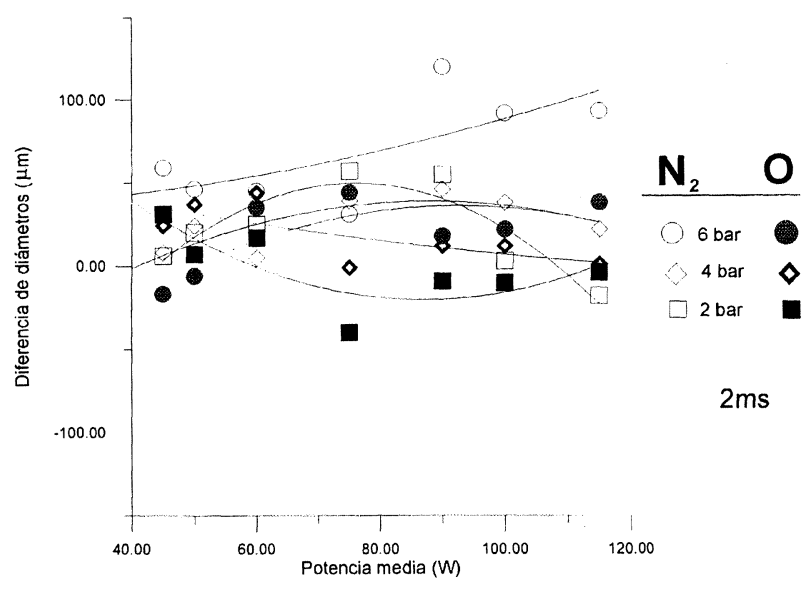

FIG. 2.- Diferencia entre el diámetro de entrada y el de salida en función de la potencia media del láser para diferentes presiones $\left(\mathrm{N}_{2}, \mathrm{y}_{2}\right)$.

FIG. 2.-Difference between inlet and outlet diameter vs average power for different pressures $\left(\mathrm{N}_{2} y_{2} \mathrm{O}_{2}\right)$. 


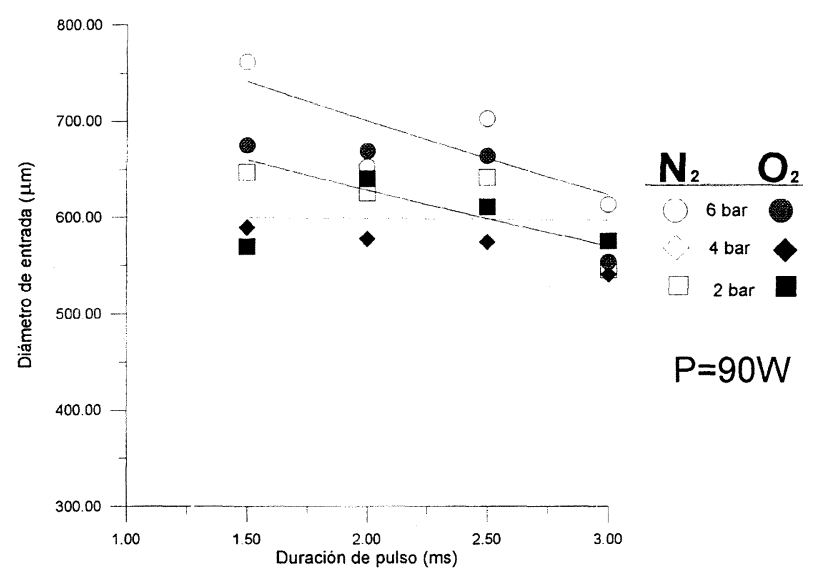

FIG. 3.- Diámetro de entrada en función de la duración de pulso, para diferentes presiones $\left(\mathrm{N}_{2}\right.$ y $\left.\mathrm{O}_{2}\right)$.

FIG. 3.- Inlet diameter vs pulse width, for different pressures $\left(\mathrm{N}_{2}\right.$ y $\left.\mathrm{O}_{2}\right)$.

obtenidos con $\mathrm{N}_{2}$ y $\mathrm{O}_{2}$ para diferentes presiones. Se puede inferir la tendencia general de que el diámetro de entrada decrece con el aumento del ancho del pulso, independientemente de la presión y del tipo de gas. Sin embargo, el papel del tipo de gas y la presión vienen siendo un factor marcadamente significativo con una duración de pulso y potencia bajos.

La figura 4 muestra el aspecto de los orificios de entrada taladrados en láminas de pizarra; estas imágenes suponen una especie de resumen de la tendencia principal observada en el presente estudio. El diámetro de entrada del orificio decrece con el aumento de la presión del gas de aportación (para ambos gases utilizados); dicha tendencia es más apreciable en el oxígeno.

Los diámetros de entrada de los orificios son más pequeños cuando se realiza el taladrado asistido por oxígeno que cuando se utiliza nitrógeno. Este comportamiento es más significativo para

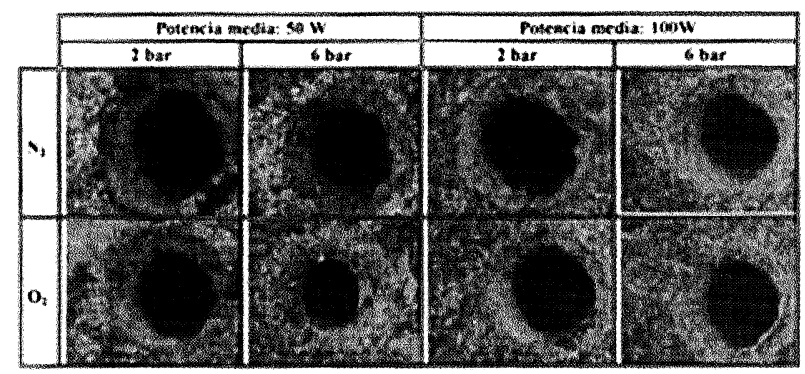

FIG. 4.- Fotografía óptica en la que se muestra el aspecto de los agujeros desde la entrada, para diferentes potencias y presiones $\left(\mathrm{N}_{2}\right.$ y $\left.\mathrm{O}_{2}\right)$.

FIG. 4.- Optical photograph showing the aspect of the holes from the inlet side for different pressures and laser power $\left(\mathrm{N}_{2} \mathrm{y}_{2} \mathrm{O}_{2}\right.$. potencias bajas $(50 \mathrm{~W})$ y presiones altas $(6$ bar). Finalmente, un aumento de la potencia del láser supone un aumento del diámetro del orificio.

\section{CONCLUSIONES}

Se ha llevado a cabo un estudio sistemático sobre la influencia de diferentes parámetros en la forma de orificios realizados por un láser de Nd:YAG sobre láminas de pizarra.

Se ha desarrollado un estudio exhaustivo de los parámetros del láser, tales como el ancho de pulso, la potencia media, además de los parámetros del gas, como, presión y tipo de gas.

Se han corroborado las ventajas (9) del uso de un láser de potencia pulsado de Nd:YAG para el taladrado de pequeños orificios en láminas de pizarra, que se pueden resumir de la manera siguiente:

- Los orificios se pueden localizar con precisión.

- El proceso de taladrado es extremadamente rápido.

- El coste de la operación es bajo.

- Por regla general, el uso del oxígeno como gas de aportación conduce a orificios de diámetro más pequeño que cuando se usa nitrógeno.

- La mejor proporción de orificios se ha obtenido para un ancho de pulso intermedio de 2 ms cuando se usa oxígeno a presión moderada de 4 bar.

- Teniendo en cuenta su flexibilidad, el proceso puede ser automatizado con facilidad.

\section{Agradecimiento}

Los autores agradecen la valiosa ayuda que les han brindado S. Chiussi, P. González, F. García Sanz y J. Bugarín.

\section{REFERENCIAS}

(1) Menéndez Seigas, J.L. Arquitectura y tecnología de la colocación de pizarra en cubiertas. Peymar Artes Gráficas, S.L. O Barco (Ourense, España). 1993.

(2) Roc Maquina Staff. Natural Stones Directory. (Bilbao, España). 1996.

(3) H. Malllet. Le Laser. Principes et techniques d'application. Edit. Lavoisier. 1984.

(4) Yukitaka Nagano, en Industrial Laser Handbook. (Eds. D. Belforte y M. Levitt). Penn Well Books, Tulsa, (Okla. EE.UU.) 1978.

(5) Patente española P9800391 (en trámite).

(6) SonA, A. en Applied Laser Tooling. (Eds. M. Pérez-Amor y O.D.D. Soares) Nijhoff, Boston (Mass. EE.UU.) 1987: 105.

(7) Powell, J. $\mathrm{CO}_{2}$ laser cutting. Springer-Verlag. Londres. 1993.

- (8) Yeo, C.Y, Tam, S.C. Jana, S. y LaU, M.W.S. J. Mater. Proc. Tech. 42, 1994: 15.

(9) Steen, W. Laser Materials Processing. North Holland, (EE.UU.) 1983. 